\title{
PTEN downregulation induces apoptosis and cell cycle arrest in uterine cervical cancer cells
}

\author{
$\mathrm{JIN}_{\text {WOO SHIN }}{ }^{*}$, SE-HEE KIM $^{2 *}$ and JIN YOUNG YOON ${ }^{2}$ \\ ${ }^{1}$ Department of Obstetrics and Gynecology, Gil Medical Center, College of Medicine, Gachon University; \\ ${ }^{2}$ Gachon Medical Research Institute, Gil Medical Center, Gachon University, Incheon 21565, Republic of Korea
}

Received November 9, 2020; Accepted June 18, 2021

DOI: 10.3892/etm.2021.10534

\begin{abstract}
The tumor suppressors PTEN and p53 are often downregulated in various human cancer types, which has been associated with a poor prognosis. Recent evidence implies that PTEN downregulation may induce growth arrest of kidney cells and cancer cells. In the present study, the role of PTEN in the proliferation and survival of cervical cancer cells was investigated. It was found that PTEN silencing promoted apoptosis and cell-cycle arrest, accompanied by a significant decrease in the proportion of cells in the S1 phase of the cell cycle. Moreover, PTEN silencing in cervical cancer cells increased levels of p53, p27, p21, phospho-ERK and cleaved caspase-3, and decreased levels of cyclin A2 and cyclin D1. Furthermore, PTEN knockdown significantly impacted the viability of cervical cancer cells. P53 silencing did not affect the ability of PTEN knockdown to induce apoptosis in cervical cancer cells. Taken together, the present study results imply that PTEN silencing induces apoptosis and decreases proliferation in cervical cancer cells; hence, PTEN inhibition may represent a promising strategy for the treatment of cervical cancer.
\end{abstract}

\section{Introduction}

Uterine cervical cancer is the fourth most common cancer and the leading cause of death from cancer among women worldwide (1). Although cervical cancer incidence and mortality rates have decreased in recent years because of screening programs and early detection of preinvasive cervical lesions, cervical cancer remains the second most common female cancer and the third leading cause of cancer-associated death among women. The mortality rate of cervical cancer is exceptionally high in

Correspondence to: Professor Jin Woo Shin, Department of Obstetrics and Gynecology, Gil Medical Center, College of Medicine, Gachon University, 774 Namdongdae-ro, Incheon 21565, Republic of Korea

E-mail: jwshin@gilhospital.com

*Contributed equally

Key words: PTEN, apoptosis, p53, cervical cancer developing countries (1). The standard of care for patients with recurrent cervical cancer is platinum-based chemotherapy; however, chemotherapy is mostly palliative, and new targeted therapeutic agents are urgently needed (2).

Phosphatase and tensin homolog deleted in chromosome 10 (PTEN) is an important tumor suppressor that is frequently mutated or downregulated in various human cancer types (3-9). PTEN mutation or downregulation can significantly enhance carcinogenesis and worsen treatment outcomes (3-9). In a recent study, in contrast to previous reports, high PTEN expression was associated with a worse prognosis in patients with wild-type p53 breast cancer (10).

PTEN and p53 interact closely, with PTEN regulating p53 function and p53 enhancing PTEN transcription (11). In PTEN-/-mice, the loss of PTEN dramatically decreased p53 protein levels (12). Nevertheless, acute loss of PTEN increased the levels and enhanced the function of p53 in prostate cancer cells (13). In a xenograft model of prostate cancer, long-term PTEN inhibition with the water-soluble vanadium-based complex VO-OHpic significantly decreased the tumor burden and prolonged the survival of tumor-bearing mice (14). Furthermore, PTEN downregulation in renal epithelial cells resulted in dedifferentiation and growth arrest (15). These anti-proliferative effects of PTEN silencing were p53-dependent (15).

However, the role of PTEN in cervical cancer remains unclear. In the present study, the effects of PTEN silencing on the proliferation, apoptosis and cell cycle of cervical cancer cells were investigated. The mechanisms underlying the effects of PTEN downregulation on the proliferation of cervical cancer cells were also explored.

\section{Materials and methods}

Cell lines and cultures. The human cervical cancer cell lines HeLa and CaSki (Korean Cell Line Bank) were used. Both cell lines were cultured in RPMI-1640 (Gibco; Thermo Fisher Scientific, Inc.) containing $10 \%$ (v/v) fetal bovine serum (FBS, Welgene, Inc.), $1 \%$ (v/v) penicillin and streptomycin. Cells were maintained in a humidified incubator with $5 \% \mathrm{CO}_{2}$ at $37^{\circ} \mathrm{C}$.

Chemicals and reagents. To minimize the possibility of cross-reactivity, cells were transfected with two different small interfering (si)RNAs targeting PTEN: siRNA1, CCA GUC AGA GGC GCU AUG UdTdT; and siRNA2, CAA GAU GUU 
UGA AAC UAU (16,17). A p53-targeting siRNA (CUA CUU CCU GAA AAC GdTdT) was also used (18). A commercial scrambled siRNA was used as a negative control (Bioneer Corporation). Lipofectamine RNAiMAX (Invitrogen; Thermo Fisher Scientific, Inc.) was used as the transfection reagent. Opti-MEM (minimal essential medium; Gibco; Thermo Fisher Scientific, Inc.) was used to prepare the siRNA-lipid complexes. For cell cycle analyses, a FITC BrdU Flow kit (BD PharMingen; BD Biosciences) was used. An APO-BrdU TUNEL Assay kit (Invitrogen; Thermo Fisher Scientific, Inc.) was used to assess apoptosis. An Annexin V-FITC kit (BD PharMingen; BD Biosciences) was used to visualize apoptotic cells under a fluorescence microscope. An EZ-cyTox kit (DoGenBio) was used to evaluate cell viability. The following primary antibodies were used: PTEN (1:1,000; cat. no. SC-6817-R; Santa Cruz Biotechnology, Inc.), cyclin D1 (1:1,000; cat. no. SC-718; Santa Cruz Biotechnology, Inc.), p53 (1:1,000; cat. no. SC-126; Santa Cruz Biotechnology, Inc.), actin (1:5,000; cat. no. SC-1615; Santa Cruz Biotechnology, Inc.), p27 (1:1,000; cat. no. 3686; Cell Signaling Technologies, Inc.), p21 (1:1,000; cat. no. 2947; Cell Signaling Technologies, Inc.), cyclin dependent kinase (cdk)-6 (1:1,000; cat. no. 3136; Cell Signaling Technologies, Inc.), cyclin E (1:1,000; cat. no. 4129; Cell Signaling Technologies, Inc.), cyclin A $(1: 1,000$; cat. no. 4656; Cell Signaling Technologies, Inc.), cyclin B1 (1:1,000; cat. no. 4138; Cell Signaling Technologies, Inc.), PARP (1:1,000; cat. no. 9542; Cell Signaling Technologies, Inc.), caspase-3 (1:1,000; cat. no. 9665; Cell Signaling Technologies, Inc.), AKT (1:2,000; cat. no. 4691; Cell Signaling Technologies, Inc.), pAKT (Thr308) (1:1,000; cat. no. 13038; Cell Signaling Technologies, Inc.), ERK1/2 (1:1,000; cat. no. 9122; Cell Signaling Technologies, Inc.), pERK1/2 (1:1,000; cat. no. 4370, Cell Signaling Technologies, Inc.), Bax (1:1,000; cat. no. 5023; Cell Signaling Technologies, Inc.), and MDM2 (1:1,000; cat. no. ab16895; Abcam). Goat anti-mouse IgG-HRP $(1: 5,000$; cat. no. sc-2005) and mouse anti-rabbit IgG-HRP (1:5,000; cat. no. sc-2357) secondary antibodies were purchased from Santa Cruz Biotechnology, Inc.

PTEN downregulation by siRNA. To synchronize the cell cycle, HeLa and CaSki cells were cultured in $100-\mathrm{mm}$ culture dishes $\left(1 \times 10^{6}\right.$ cells/dish) under serum-starvation and antibiotic-free conditions. After $48 \mathrm{~h}$, the cell culture medium was refreshed and the cells were maintained under serum-starvation and antibiotic-free conditions. Cells were transfected with siRNAs targeting PTEN and a negative control using the Lipofectamine RNAiMAX transfection reagent according to the manufacturer's instructions. Briefly, siRNA-lipid complexes were prepared in $500 \mu$ l Opti-MEM media by mixing $20 \mu \mathrm{l}$ transfection reagent and $20 \mu \mathrm{M}$ siRNAs (PTEN and negative control) for $5 \mathrm{~min}$ at room temperature. The mixtures were added to the cells in $10 \mathrm{ml}$ culture medium. After $6 \mathrm{~h}$ of transfection, the medium was replaced with RPMI-1640 containing 10\% (v/v) FBS, $1 \%$ (v/v) penicillin and streptomycin. Cells were incubated in complete growth medium for $48 \mathrm{~h}$.

Western blot analysis. Transfected cells were harvested and lysed in RIPA buffer supplemented with protease inhibitors. Protein concentration was determined using BCA protein assay (Thermo Fisher Scientific, Inc.). After incubation for $30 \mathrm{~min}$ on ice, cell lysates containing 10-30 $\mu \mathrm{g}$ protein per well were resolved by 10-12\% SDS-PAGE and transferred onto polyvinylidene difluoride membranes. The membranes were blocked with $5 \%(\mathrm{w} / \mathrm{v})$ nonfat dried skimmed milk in Tris-buffered saline containing $0.1 \%$ Tween-20 (pH 7.6) overnight at $4^{\circ} \mathrm{C}$ with agitation. Subsequently, membranes were incubated with primary antibodies overnight at $4^{\circ} \mathrm{C}$ with agitation. After three washes with $1 \mathrm{X}$ Tris-buffered saline containing $0.1 \%$ Tween-20, the membranes were incubated with horseradish peroxidase-conjugated secondary antibodies $(1: 5,000)$ for $1 \mathrm{~h}$ at room temperature. Signals were developed with a chemiluminescence reagent (Advansta); chemiluminescence was detected using an Amersham Imager 600 (GE Healthcare Biosciences AB).

Flow cytometry. Bromodeoxyuridine (BrdU) incorporation analysis was conducted to evaluate cell-cycle progression. Briefly, serum-starved siRNA-transfected cells $\left(5 \times 10^{5}\right.$ cells in $100-\mathrm{mm}$ culture dish) were cultured in complete growth media for $48 \mathrm{~h}$ and then labeled with $10 \mu \mathrm{M} \mathrm{BrdU}$ at $37^{\circ} \mathrm{C}$ for $60 \mathrm{~min}$. After labeling with $\mathrm{BrdU}$, cells were harvested and immunostained with FITC-conjugated anti-BrdU according to the manufacturer's instructions. BrdU incorporation was evaluated by flow cytometry (BD-FACSCalibur; BD Biosciences).

Apoptosis was assessed by measuring the amount of 5-bromo-2-deoxyuridine 5-triphosphate (BrdUTP) incorporated into the DNA using flow cytometry. Transfected cells were harvested, fixed with paraformaldehyde [1\% (w/v) in PBS], and labeled with $8.0 \mu \mathrm{l}$ BrdUTP (APO-BrdU TUNEL Assay kit) and $31.25 \mu 1 \mathrm{H}_{2} \mathrm{O}$ according to the manufacturer's instructions. Stained cells were analyzed on a BD-FACSCalibur, (BD Biosciences).

Visualization of apoptotic cells by fluorescence microscopy. To confirm the role of PTEN silencing in apoptosis, Annexin V-FITC staining was performed, followed by fluorescence microscopy (magnification, x200). Briefly, transfected HeLa cells were washed twice with PBS and once with Annexin V binding buffer. Subsequently, cells were stained with Annexin V-FITC (1:10 in Annexin V binding buffer) for $15 \mathrm{~min}$ at room temperature. Cells were washed and observed under a confocal microscope (LSM 700; Carl Zeiss AG). ImageJ software was used for quantification.

MTT assay. The viability of HeLa and CaSki cells was analyzed following transfection with PTEN and control siRNAs. Briefly, siRNA-transfected cells were incubated with water-soluble tetrazolium salts $(1 / 10$ of the volume of the culture medium; EZ-cyTox, DoGenBio) at $37^{\circ} \mathrm{C}$ for $2 \mathrm{~h}$. Optical absorbance at $450 \mathrm{~nm}$ was measured using a microplate reader. The optical absorbance of the culture medium alone was measured as blank.

Statistical analysis. Data were expressed as mean \pm standard deviation. All experiments were performed more than three times. Independent-t tests and Mann-Whitney U tests were performed using SPSS 17.0 (SPSS, Inc.). $P<0.05$ was considered to indicate a statistically significant difference. 


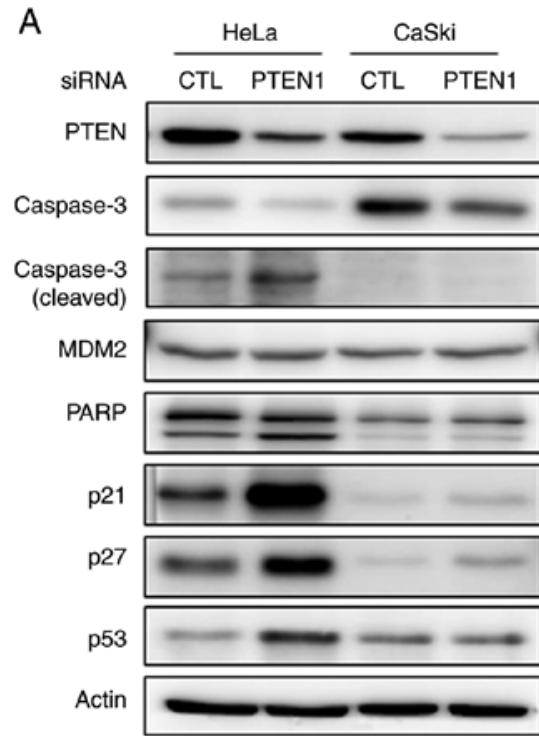

B

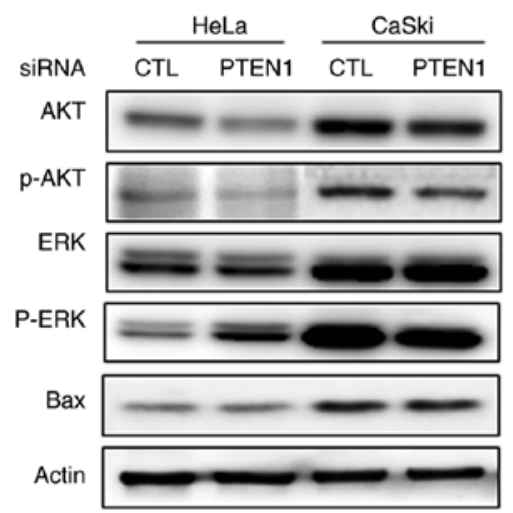

C

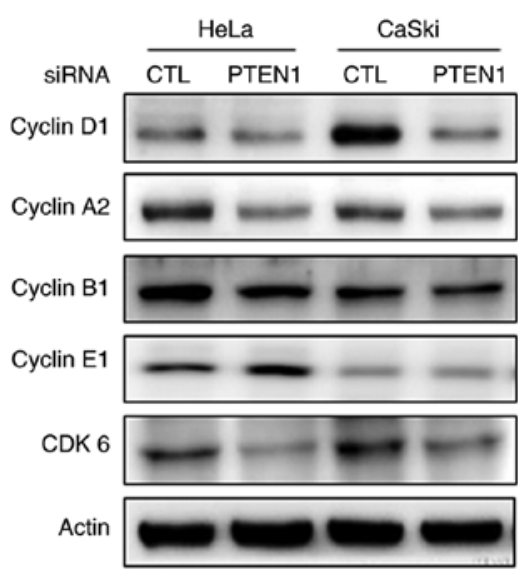

D

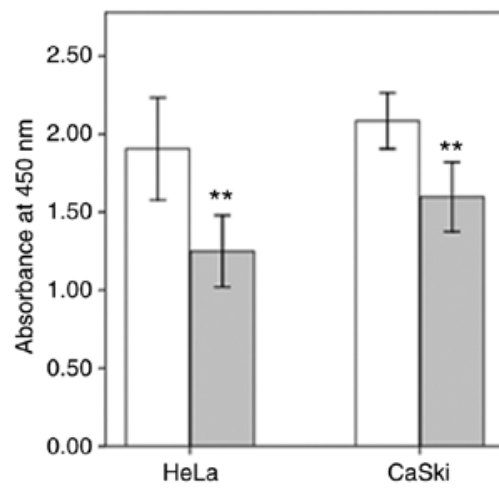

SiRNA

$\square$ Control siRNA

口PTEN SIRNA1

Figure 1. PTEN silencing regulates the expression levels of proteins involved in apoptosis and the cell cycle. (A-C) Western blots showing the levels of proteins involved in apoptosis (caspase-3, MDM2, PARP, p21, p27, p53 and Bax), cell cycle (cyclin D1, cyclin A2, cyclin B1, cyclin E1 and CDK6), and AKT and ERK signaling pathways in cervical cancer cells transfected with PTEN siRNA1. (D) Cell viability of HeLa and CaSki cells transfected with PTEN-targeting and control siRNAs (error bars represent $95 \%$ confidence intervals). ${ }^{* *} \mathrm{P}<0.01$ vs. control siRNA. siRNA, small interfering RNA; CTL, control siRNA; PTEN1, PTEN SiRNA1.

\section{Results}

PTEN downregulation enhances the expression of apoptosisassociated proteins. In order to assess the effects of PTEN downregulation on the expression levels of proteins involved in cellular fate, western blotting was performed to measure the levels of proteins associated with cellular apoptosis and senescence. Notably, PTEN silencing in HeLa cells increased the levels of p53 and p53-associated proteins (Fig. 1A). Although PTEN silencing in CaSki cells did not alter the levels of p53, levels of p21 and p27 were increased in PTEN siRNA-transfected cells.

p53 is regulated by the extracellular signal regulated kinase (ERK) and is essential for the activation of downstream ERK pathway components. Activation of caspase-3 and poly (ADP-ribose) polymerase (PARP) by ERK induces apoptosis $(19,20)$. PARP is a substrate for caspase-3, and PARP cleavage promotes apoptosis by inhibiting DNA repair (21). In the study, PTEN silencing in HeLa cells increased the levels of phosphorylated (p)ERK, p53, caspase-3 and cleaved PARP; Bax, levels remained unchanged (Fig. 1B). It was also investigated whether PTEN downregulation affects cell proliferation and viability. Indeed, PTEN silencing inhibited the proliferation of HeLa and CaSki cells, implying that PTEN downregulation induces apoptosis in cervical cancer cells (Fig. 1D).

PTEN downregulation represses the expression of cell cycle-associated proteins. To achieve cellular senescence, an increased amount of Cdk inhibitor p21, a target of p53, can inactivate both cyclin $\mathrm{E}$ and cyclin D1-associated kinase $(22,23)$.

In order to examine the role of PTEN in cell-cycle regulation in cervical cancer cells, the levels of cell-cycle-associated proteins were analyzed after PTEN silencing. The levels of Cdk6, cyclin D1 and cyclin A2 were decreased after PTEN silencing (Fig. 1C). However, PTEN downregulation did not alter the levels of cyclin E1 and cyclin B1 in HeLa or CaSki cells (Fig. 1C).

Furthermore, flow cytometry analyses revealed that the proportion of cells in the $G_{0} / G_{1}$ and sub- $G_{1}$ phases was increased after PTEN silencing. On the other hand, the proportion of cells in the S-phase was decreased when PTEN was downregulated (Figs. 2 and S1). These findings imply that PTEN downregulation promotes overexpression of p53 and p21, and causes apoptosis and $\mathrm{G}_{1}$ cell-cycle arrest in HeLa cells $(4,13,15,24)$. 
A

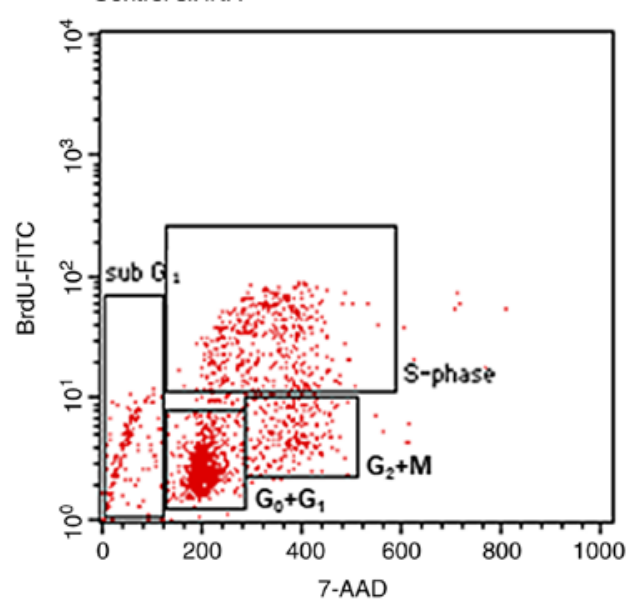

B PTEN SIRNA 1

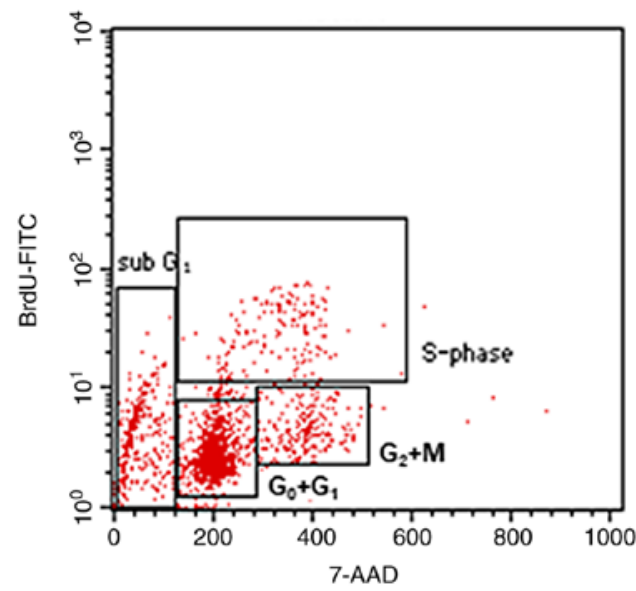

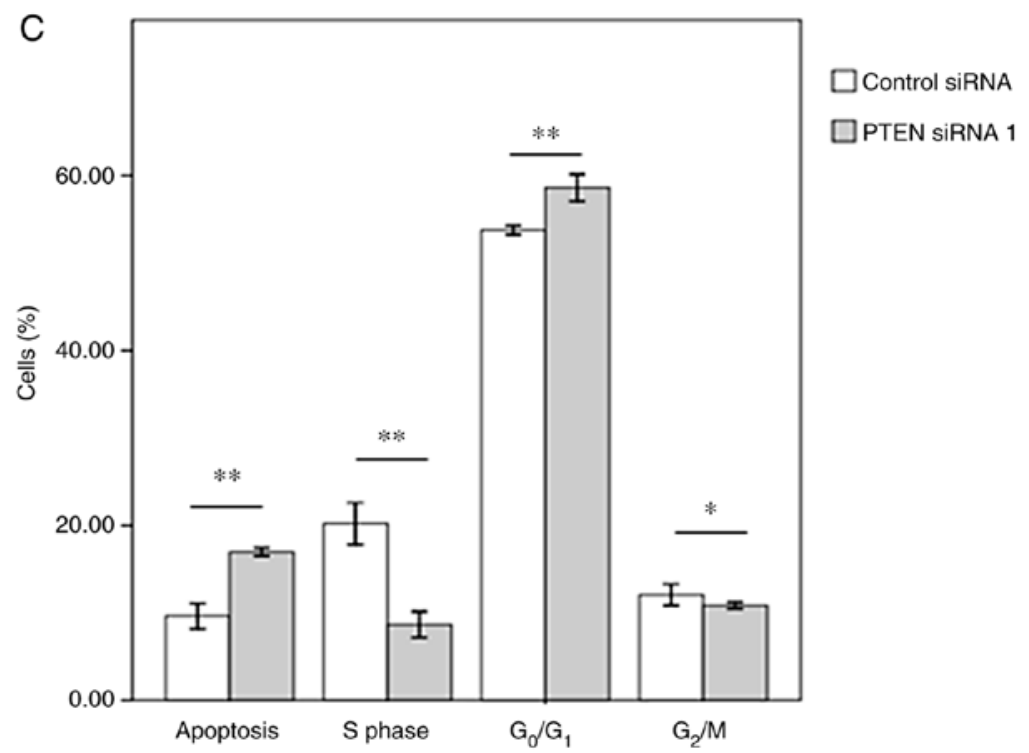

Figure 2. PTEN silencing regulates cell-cycle progression in HeLa cells. The cell-cycle phase in control (A) and PTEN siRNA1-transfected (B) cells. (C) Mean percentage of the cell population in each cell-cycle phase (error bars represent $95 \%$ confidence intervals). " $\mathrm{P}<0.05$ and ${ }^{* *} \mathrm{P}<0.01$ vs. control siRNA. siRNA, small interfering RNA.

PTEN downregulation induces apoptosis independently of $p 53$. In order to confirm the role of PTEN in the apoptosis of cervical cancer cells, TUNEL assay and flow cytometry were performed using PTEN siRNA-transfected HeLa cells. As shown in Fig. 3A and B, PTEN silencing increased the proportion of HeLa cells undergoing apoptosis. To investigate the role of p53 in the apoptosis of cells with PTEN downregulation, HeLa cells were co-transfected with siRNAs targeting PTEN and p53. Cells co-transfected with PTEN-targeting and scrambled siRNAs were used as a control. PTEN promoted apoptosis in cervical cancer cells regardless of the levels of p53 (Fig. 3B and C). These results imply that p53 is not essential for the induction of apoptosis in HeLa cells in response to PTEN downregulation.

\section{Discussion}

Regulation of DNA damage during the cell cycle is essential for cell survival. Cells with unrepaired DNA damage undergo apoptosis or cell-cycle arrest due to the activation of DNA-damage-response pathways (25).
PTEN and p53 are crucial for DNA damage repair and the maintenance of genomic integrity (26). Although PTEN and p53 are often downregulated in cervical cancer, somatic mutations in PTEN are rare, ranging from 0 to 2\% (7,27); p53 mutations have been reported in $13.3 \%$ of cervical adenocarcinomas and $5.9 \%$ of cervical squamous cell carcinomas (28). Although downregulation of PTEN and p53 has been reported in cervical cancer $(8,29)$, no somatic mutations in these genes were detected in HeLa and CaSki cells $(30,31)$, allowing cancer cells to survive despite having unresolved DNA damage. In the present study, PTEN downregulation promoted apoptosis and cell-cycle arrest in cervical cancer cells (Figs. 1 and 2),implying that loss of PTEN impairs the ability of cells to repair DNA damage. Unrepaired DNA damage in cervical cancer cells may promote apoptosis by activating DNA-damage-response pathways.

The PTEN, PI3K and AKT signaling pathways are critical regulators of cell proliferation and apoptosis $(3,32)$. The downregulation of PTEN is associated with the activation of PI3K and AKT signaling, cell proliferation and a worse prognosis 

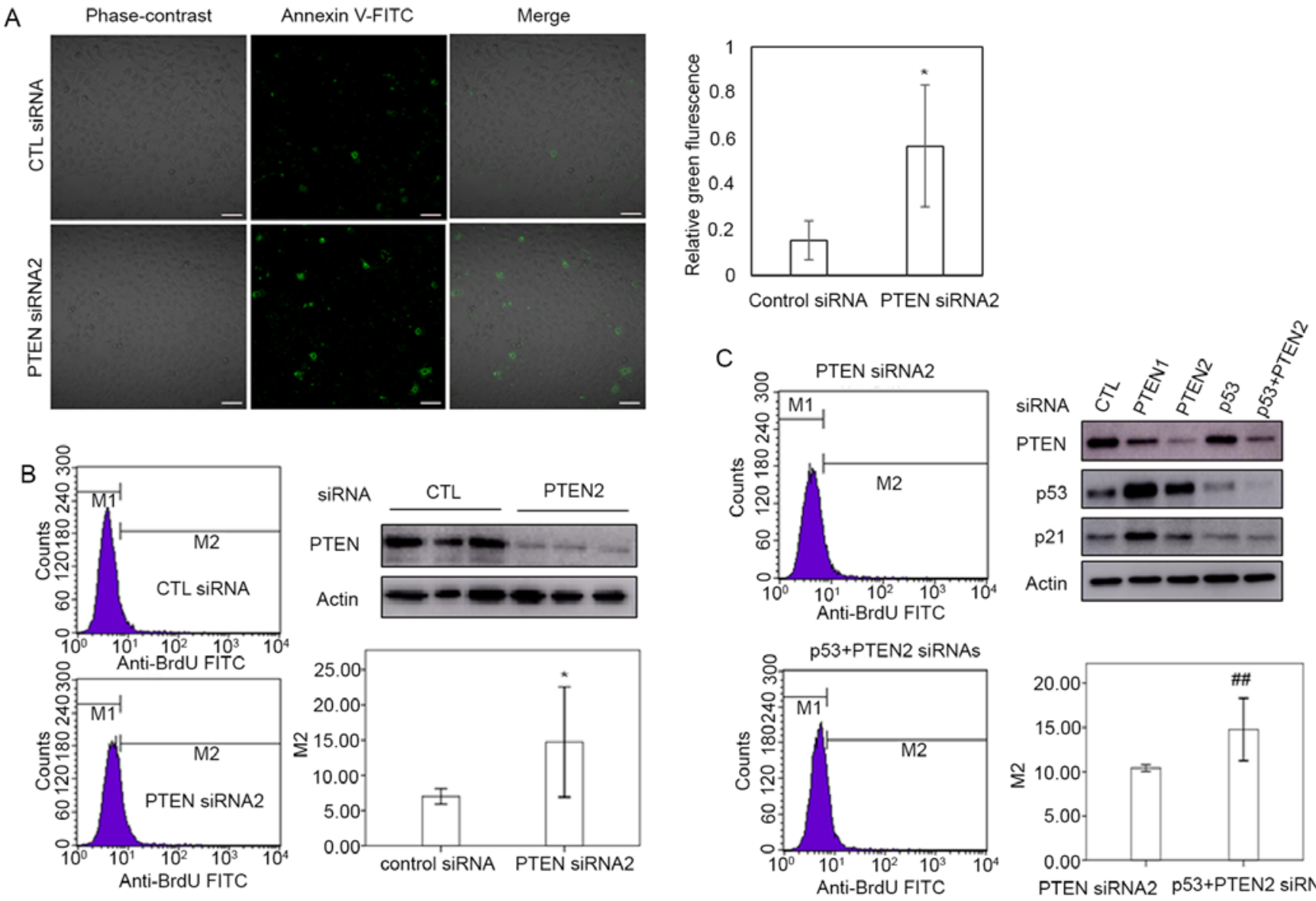

Figure 3. PTEN downregulation induces apoptosis independently of p53 in HeLa cells. (A) Apoptotic cells were visualized by fluorescence microscopy. Cells transfected with control and PTEN siRNA2 were fixed and stained with Annexin V-FITC. The graph shows quantitative analysis of relative Annexin V fluorescence intensity. ImageJ software was used for quantification. (B) Flow cytometry analysis of control and PTEN siRNA2-transfected cells. PTEN expression in control and PTEN siRNA2-transfected cells was evaluated by western blotting. The graph shows the percentage of BrdU staining in control and PTEN siRNA-transfected cells. (C) Flow cytometry analysis of cells transfected with control siRNA + PTEN siRNA2 or PTEN siRNA2 + p53 siRNA (error bars represent $95 \%$ confidence intervals). Western blots showing the expression levels of PTEN, p53, p21 and actin. The graph shows the percentage of BrdU-positive cells in the control + PTEN siRNA2 and p53 + PTEN siRNA2 groups. ${ }^{*} \mathrm{P}<0.05$ vs. control siRNA; ${ }^{\#} \mathrm{P}<0.01$ vs. control siRNA + PTEN siRNA2. Scale bar, $50 \mu \mathrm{m}$. siRNA, small interfering RNA; CTL, control siRNA; C, control siRNA; PTEN1, PTEN siRNA1; PTEN2, PTEN siRNA2.

in various cancer types (3-5). However, Tan and Chiu (20) reported that the activation of ERK inhibited the AKT pathway. In the present study, loss of PTEN activated the ERK pathway; nevertheless, the expression levels of MDM2 and phospho-AKT were unchanged regardless of the PTEN status in HeLa cells (Fig. 1A and B).This implies that PTEN regulates p53 levels independently of MDM2 and AKT, consistent with a previous report (12). In contrast to the present observations in HeLa cells, PTEN silencing did not affect AKT activation or MDM expression in CaSki cells. Thus, the regulation of the PTEN-ERK-p53-PARP axis may differ depending on cell type.

Although the loss of both PTEN and p53 enhances tumor aggressiveness, the inactivation of PTEN with intact p53 induces cellular senescence (13). Similar regulatory mechanisms have been reported for BRCA1/2 and p53 in breast cancer $(33,34)$. Furthermore, PTEN inhibition in hepatocarcinoma cells expressing low levels of PTEN has been shown to drive senescence (35). Although PTEN downregulation is common in cervical cancer $(8,9)$, somatic mutations in PTEN are rare $(7,27)$. Therefore, most cervical cancer have intact PTEN, but expressed at low levels, similar to reports regarding hepatocarcinoma (35). In the present study, PTEN inhibition in cervical cancer cells expressing low levels of PTEN promoted cellular senescence and apoptosis, which was independent of p53 status. Therefore, PTEN downregulation in cervical cancer may result in p53 upregulation, thereby inducing apoptosis in cervical cancer cells (Fig. 1).

In conclusion, the present data imply that PTEN downregulation in cervical cancer cells induces cell-cycle arrest and apoptosis. Furthermore, decreased cell viability was observed as a result of PTEN downregulation in cervical cancer cells. Taken together, the present study findings may present a possible strategy for cervical cancer treatment via the regulation of PTEN. More studies will need to follow that will not only detail the effects of PTEN regulation but also reinforce some of the limitations of the present study, which include lack of various forms of analysis and clear mechanistic study. The limitations of the present study will be addressed in a subsequent study, which will mainly focus on elucidating the role of p53 in cervical cancer cell apoptosis by checking gene expression levels of components that are essential for apoptosis induced by PTEN downregulation. 


\section{Acknowledgements}

Not applicable.

\section{Funding}

This work was supported by the Gil Medical Center, Gachon University (grant no. 2012-03).

\section{Availability of data and materials}

The datasets used and/or analyzed during the current study are available from the corresponding author on reasonable request.

\section{Authors' contributions}

JWS and JYY conducted the experiments and analyzed the results. JWS designed the study and wrote the manuscript. SHK performed analysis and interpretation of data, and the overall editing of the manuscript. JYY and SHK confirmed the authenticity of all the raw data. All the authors have read and approved the final version of the manuscript.

\section{Ethics approval and consent to participate}

Not applicable.

\section{Patient consent for publication}

Not applicable.

\section{Competing interests}

The authors declare that they have no competing interests.

\section{References}

1. Torre LA, Bray F, Siegel RL, Ferlay J, Lortet-Tieulent J and Jemal A: Global cancer statistics, 2012. CA Cancer J Clin 65: 87-108, 2015.

2. Boussios S, Seraj E, Zarkavelis G, Petrakis D, Kollas A, Kafantari A, Assi A, Tatsi K, Pavlidis N and Pentheroudakis G: Management of patients with recurrent/advanced cervical cancer beyond first line platinum regimens: Where do we stand? A literature review. Crit Rev Oncol Hematol 108: 164-174, 2016.

3. Liu YZ, Wu K, Huang J, Liu Y, Wang X, Meng ZJ, Yuan SX Wang DX, Luo JY, Zuo GW, et al: The PTEN/PI3K/Akt and $\mathrm{Wnt} / \beta$-catenin signaling pathways are involved in the inhibitory effect of resveratrol on human colon cancer cell proliferation. Int J Oncol 45: 104-112, 2014.

4. Kim J, Eltoum IE, Roh M, Wang J and Abdulkadir SA: Interactions between cells with distinct mutations in c-MYC and Pten in prostate cancer. PLoS Genet 5: e1000542, 2009.

5. Yanagawa N, Leduc C, Kohler D, Saieg MA, John T, Sykes J, Yoshimoto M, Pintilie M, Squire J, Shepherd FA and Tsao MS Loss of phosphatase and tensin homolog protein expression is an independent poor prognostic marker in lung adenocarcinoma. J Thorac Oncol 7: 1513-1521, 2012.

6. Kandoth C, McLellan MD, Vandin F, Ye K, Niu B, Lu C, Xie M, Zhang Q, McMichael JF, Wyczalkowski MA, et al: Mutational landscape and significance across 12 major cancer types. Nature 502: 333-339, 2013.

7. Tashiro H, Blazes MS, Wu R, Cho KR, Bose S, Wang SI, Li J, Parsons R and Ellenson LH: Mutations in PTEN are frequent in endometrial carcinoma but rare in other common gynecological malignancies. Cancer Res 57: 3935-3940, 1997.
8. Lu D, Qian J, Yin X, Xiao Q, Wang C and Zeng Y: Expression of PTEN and survivin in cervical cancer: Promising biological markers for early diagnosis and prognostic evaluation. Br J Biomed Sci 69: 143-146, 2012.

9. Lee JS, Choi YD, Lee JH, Nam JH, Choi C, Lee MC, Park CS, Kim HS and Min KW: Expression of PTEN in the progression of cervical neoplasia and its relation to tumor behavior and angiogenesis in invasive squamous cell carcinoma. J Surg Oncol 93: 233-240, 2006

10. Yndestad S, Austreid E, Knappskog S, Chrisanthar R, Lilleng PK, Lønning PE and Eikesdal HP: High PTEN gene expression is a negative prognostic marker in human primary breast cancers with preserved p53 function. Breast Cancer Res Treat 163: 177-190, 2017.

11. Nakanishi A, Kitagishi Y, Ogura Y and Matsuda S: The tumor suppressor PTEN interacts with p53 in hereditary cancer (Review). Int J Oncol 44: 1813-1819, 2014.

12. Blanco-Aparicio C, Renner O, Leal JF and Carnero A: PTEN, more than the AKT pathway. Carcinogenesis 28: 1379-1386, 2007.

13. Chen Z, Trotman LC, Shaffer D, Lin HK, Dotan ZA, Niki M, Koutcher JA, Scher HI, Ludwig T, Gerald W, et al: Crucial role of p53-dependent cellular senescence in suppression of Pten-deficient tumorigenesis. Nature 436: 725-730, 2005.

14. Alimonti A, Nardella C, Chen Z, Clohessy JG, Carracedo A, Trotman LC, Cheng K, Varmeh S, Kozma SC, Thomas G, et al: A novel type of cellular senescence that can be enhanced in mouse models and human tumor xenografts to suppress prostate tumorigenesis. J Clin Invest 120: 681-693, 2010.

15. Samarakoon R, Helo S, Dobberfuhl AD, Khakoo NS, Falke L, Overstreet JM, Goldschmeding R and Higgins PJ: Loss of tumour suppressor PTEN expression in renal injury initiates SMAD3- and p53-dependent fibrotic responses. J Pathol 236: 421-432, 2015.

16. Gupta A and Dey CS: PTEN and SHIP2 regulates PI3K/Akt pathway through focal adhesion kinase. Mol Cell Endocrinol 309: 55-62, 2009.

17. Mise-Omata S, Obata Y, Iwase S, Mise N and Doi TS: Transient strong reduction of PTEN expression by specific RNAi induces loss of adhesion of the cells. Biochem Biophys Res Commun 328: 1034-1042, 2005.

18. Scacheri PC, Rozenblatt-Rosen O, Caplen NJ, Wolfsberg TG, Umayam L, Lee JC, Hughes CM, Shanmugam KS, Bhattacharjee A, Meyerson M and Collins FS: Short interfering RNAs can induce unexpected and divergent changes in the levels of untargeted proteins in mammalian cells. Proc Natl Acad Sci USA 101: 1892-1897, 2004.

19. Li DW, Liu JP, Mao YW, Xiang H, Wang J, Ma WY, Dong Z, Pike HM, Brown RE and Reed JC: Calcium-activated RAF/MEK/ERK signaling pathway mediates p53-dependent apoptosis and is abrogated by alpha B-crystallin through inhibition of RAS activation. Mol Biol Cell 16: 4437-4453, 2005.

20. Tan BJ and Chiu GN: Role of oxidative stress, endoplasmic reticulum stress and ERK activation in triptolide-induced apoptosis. Int J Oncol 42: 1605-1612, 2013.

21. D'Amours D, Sallmann FR, Dixit VM and Poirier GG: Gain-of-function of poly(ADP-ribose) polymerase-1 upon cleavage by apoptotic proteases: Implications for apoptosis. J Cell Sci 114: 3771-3778, 2001.

22. Stein GH, Drullinger LF, Soulard A and Dulić V: Differential roles for cyclin-dependent kinase inhibitors p21 and p16 in the mechanisms of senescence and differentiation in human fibroblasts. Mol Cell Biol 19: 2109-2117, 1999.

23. Althubiti M, Lezina L, Carrera S, Jukes-Jones R, Giblett SM, Antonov A, Barlev N, Saldanha GS, Pritchard CA, Cain K and Macip S: Characterization of novel markers of senescence and their prognostic potential in cancer. Cell Death Dis 5: e1528, 2014.

24. Kim JS, Lee C, Bonifant CL, Ressom H and Waldman T: Activation of $\mathrm{p} 53$-dependent growth suppression in human cells by mutations in PTEN or PIK3CA. Mol Cell Biol 27: 662-677, 2007.

25. Zhou BB and Elledge SJ: The DNA damage response: Putting checkpoints in perspective. Nature 408: 433-439, 2000.

26. Ming $M$ and He YY: PTEN in DNA damage repair. Cancer Lett 319: 125-129, 2012.

27. Su TH, Chang JG, Perng LI, Chang CP, Wei HJ, Wang NM and Tsai CH: Mutation analysis of the putative tumor suppressor gene PTEN/MMAC1 in cervical cancer. Gynecol Oncol 76: 193-199, 2000 . 
28. Tornesello ML, Buonaguro L and Buonaguro FM: Mutations of the TP53 gene in adenocarcinoma and squamous cell carcinoma of the cervix: A systematic review. Gynecol Oncol 128: 442-448, 2013.

29. Qi Q, Ling Y, Zhu M, Zhou L, Wan M, Bao Y and Liu Y: Promoter region methylation and loss of protein expression of PTEN and significance in cervical cancer. Biomed Rep 2: 653-658, 2014.

30. Yaginuma $\mathrm{Y}$ and Westphal $\mathrm{H}$ : Analysis of the p53 gene in human uterine carcinoma cell lines. Cancer Res 51: 6506-6509, 1991.

31. Meric-Bernstam F, Akcakanat A, Chen H, Do KA, Sangai T, Adkins F, Gonzalez-Angulo AM, Rashid A, Crosby K, Dong M, et al: PIK3CA/PTEN mutations and Akt activation as markers of sensitivity to allosteric mTOR inhibitors. Clin Cancer Res 18: 1777-1789, 2012.

32. Chalhoub $\mathrm{N}$ and Baker SJ: PTEN and the PI3-kinase pathway in cancer. Annu Rev Pathol 4: 127-150, 2009.
33. XuX,WagnerKU,Larson D, WeaverZ,LiC,RiedT,Hennighausen L, Wynshaw-Boris A and Deng CX: Conditional mutation of Brcal in mammary epithelial cells results in blunted ductal morphogenesis and tumour formation. Nat Genet 22: 37-43, 1999.

34. Jonkers J, Meuwissen R, van der Gulden H, Peterse H, van der Valk M and Berns A: Synergistic tumor suppressor activity of BRCA2 and p53 in a conditional mouse model for breast cancer. Nat Genet 29: 418-425, 2001.

35. Augello G, Puleio R, Emma MR, Cusimano A, Loria GR, McCubrey JA, Montalto G and Cervello M: A PTEN inhibitor displays preclinical activity against hepatocarcinoma cells. Cell Cycle 15: 573-583, 2016.

This work is licensed under a Creative Commons Attribution-NonCommercial-NoDerivatives 4.0 International (CC BY-NC-ND 4.0) License. 